\title{
Phoneme Resistance and Phoneme Confusion in Noise: Impact of Dyslexia
}

\author{
Noelia Do Carmo Blanco ${ }^{1}$, Julien Meyer ${ }^{2}$, Michel Hoen ${ }^{3}$, Fanny Meunier ${ }^{1}$ \\ ${ }^{1}$ Université Côte d’Azur, CNRS, BCL, France \\ ${ }^{2}$ Université Grenoble Alpes, CNRS, GIPSA-Lab, Grenoble, France \\ ${ }^{3}$ Oticon Medical, Vallauris, France \\ ndocarmo@unice.fr, julien.meyer@gipsa-lab.fr, mhoe@oticonmedical.com, \\ fanny.meunier@unice.fr
}

\begin{abstract}
Understanding speech in noisy environments is a challenge for almost everyone and particularly so for people with dyslexia. To better understand the phonological processing deficit, which has been posited as a core trait of dyslexia, we wanted to further characterize the impact of noise on speech perception. In this paper, we investigated phoneme resistance to noise for dyslexic and control adults and explored the pattern of errors produced by noise interference. Our aim was to examine differences between phoneme confusion matrices of the two populations.

Disyllabic nouns were embedded in noise and participants had to perform an auditory word identification task. Error rates, phoneme resistance and phoneme confusions were compared between a dyslexic and a group of matched controls.

Error rate was higher in the dyslexic group. However, no qualitative differences in the profile of errors were found. The coronals $/ \int$ and $\mathrm{s} /$ were the most resistant phoneme in both groups while the labials /f, $\mathrm{m}$ and $\mathrm{v} /$ were the most vulnerable. Although dyslexics showed a more scattered pattern of confusions, the matrices were correlated. Our results confirm a phonological deficit in dyslexia whereas they do not support the hypothesis of qualitative differences in phonological representation between the two groups.
\end{abstract}

Index Terms: word identification, speech-in-noise, dyslexia, phoneme resistance, phoneme confusion

\section{Introduction}

Dyslexia is a developmental language disorder occurring in the absence of sensory or neurological impairments. It is defined as a specific impairment in the development of normal reading skills. Although the etiology of dyslexia remains controversial, it is well established that people with dyslexia show deficits in phonological processing [1]. Relatedly, it appears that dyslexia has effects not only on reading skills but also on the processing of acoustic and phonological information. In particular, it has been reported that an important proportion of dyslexics experience difficulties in understanding speech-in-noise [2], in the absence of diagnosed hearing loss, while no differences have been observed in speech perception in quiet environments between dyslexics and control subjects $[3,4]$. This speech-innoise impairment has even been proposed as a core deficit of dyslexia as indeed, behavioral measures of speech-in-noise comprehension predict reading performances better than other cognitive, auditory or attentional measures [3]. This impairment can be very disabling in daily life as most auditory communications take place in the presence of noise. The study of this deficit might shed light on the causes of the condition and might help in developing compensatory strategies.

The speech-in-noise deficit initially observed in dyslexic children is maintained at adult age and dyslexic adults also experience more difficulties understanding speech in noisy environments compared to normal-reading adults. With experience, dyslexics often develop compensatory strategies, giving sometimes the misleading impression that dyslexia disappears with age. In fact, it has been reported that on average, dyslexic adults performed less well than matched normal-readers at tasks evaluating speech perception in the presence of noise, even in experiments in which no written material was involved $[5,6]$. The first aim of this study is to replicate prior results that showed a deficit in perception of speech-in-noise in dyslexic adults.

In a previous study, we looked at phoneme recognition error patterns for unimpaired participants [7]. The overall picture shows that vowels are much more resistant than consonants and that errors on consonants were mostly confusions, whereas deletion is also a type of errors for vowels. Performed analyses showed a limited impact of noise type on phoneme intelligibility. For example, the phoneme /v/, presented a stable pattern over noise conditions as being one of the less resistant consonants and /s/ and / $/$ / the most resistant ones. Yet, the profile of phoneme errors resulting from the masking effect has never been studied for dyslexics. The second question we raise in this paper concerns differences between unimpaired subjects and dyslexics in the pattern of phoneme resistance and vulnerability to noise. It has been suggested that phonological representations are somehow degraded in dyslexia. The nature of the presumed degradation is still in debate. It includes, depending on authors, increased noise, phonetic underspecification, decreased temporal or spectral resolution, and/or less categorical representations (see [6] for an overview). If the phonological representations are degraded, we might expect fewer phonemes to be resistant to noise and more to be vulnerable.

The pattern of phoneme confusion could also shed light on phonological processing. It has been shown that confusion patterns are the result of not only acoustic properties and frequency of phonemes but also complex higher-level phonological processing [8] and thus they can be asymmetric. For instance, /v/ elicits more /ð/ responses than /ð/ elicits /v/ [9]. It has been proposed that response biases contribute to confusion patterns. By characterizing the pattern of phoneme confusions in adults with dyslexia we can examine whether the response biases correspond to those present in non-dyslexic participants. If the phonological representations in dyslexia are 
degraded or less defined, we might expect a broader pattern of phoneme confusions under noise condition.

\section{Methods}

Participants performed a speech-in-noise identification task.

\subsection{Participants}

The study included 20 adults with a documented history of dyslexia (12 females, 8 males, mean age: 24.6 years, SD: 5.79) and 20 controls (16 females, 4 males, mean age: 24.35 years, SD: 5.68) matched in age, years of study, non-verbal intellectual abilities (as assessed with the Raven's Standard Progressive Matrices) and handedness. All dyslexic participants had been diagnosed by a psychologist or neuropsychologist prior to inclusion. All participants underwent a standardized reading test which showed significant differences between groups (see scores of the different tests and statistical analysis in [5]). All participants were right handed native French speakers, aged from 18 to 45 years, and had no other documented hearing or language disorders. The study was approved by the local ethics committee (ID RCB: 2008A00708-47). Participants gave informed consent and received a monetary compensation. They were screened for audiometric pure-tone thresholds before participating in the study and no one exceeded $20 \mathrm{~dB}$ HL at any frequency between 250 and 8000 $\mathrm{Hz}$.

\subsection{Stimuli}

Stimuli for the word recognition task consisted of 126 disyllabic words embedded in noise. The words were pronounced by a 24 year-old French native female speaker. They were recorded in a sound-proof room, at a sampling rate of $44.1 \mathrm{kHz}$. Stimuli were created by embedding one target-word in a randomly selected 4 -s burst of noise, where the target words started $2.5 \mathrm{~s}$ after noise onset.

\subsubsection{Target words}

The selected target words were disyllabic nouns with a middle range of frequency of occurrence ( 0.13 to 338.19 occurrence per million (opm), average: $16.82 \mathrm{opm}, \mathrm{SD}: 43.74)$ according to the French database Lexique2 [10]. Regarding the first syllable, on which most of the analysis is focused, the set comprised $108 \mathrm{CV}$ and $18 \mathrm{CCV}$ (subsequently, the first consonant of the cluster will be named C1 and the second C1'). The occurrence of $\mathrm{C} 1$ phonemes was as follows: /p/ 18 times, /b,k/ 17 times, /в/ 12 times, / $/ 10$ times /s,t,l/ 7 times, /d,f,g,m,v,z/ 5 times and /n/ once.

\subsubsection{Noise}

Three different types of noise were used to avoid any specific effects: a cocktail-party noise (speech from a 4-talkers babble, 2 males and 2 females), a modulated noise and a stationary broadband noise (see [5] for a detailed description of the background noise and the different SNR conditions). Both, the modulated and the broadband noise were derived from the original cocktail-party sound and created by extracting the envelope information under $60 \mathrm{~Hz}$.

\subsection{Procedure}

Participants were presented with the stimuli over headphones while seated in a sound-attenuated room. Only one target word out of the 126 was played in each trial and the order of presentation was random. Participants were instructed to listen carefully and to repeat the word they heard to the experimenter. Responses were transcribed verbatim by the experimenter and audiotaped to allow to double check the transcription. Participants did not receive feedback on their performance.

\subsection{Analysis}

To compare performance on speech understanding in noise between the dyslexic and the control group of participants we computed quantitative measures (i.e. error rates) as well as qualitative measures (i.e. profile of errors, including phoneme resistance to noise and phoneme confusions). Analyses of group differences in performance (excluding phoneme analysis) were assessed using unpaired $t$-tests and mixed model analysis of variance (ANOVA) with group (dyslexics and controls) as the between-subjects factor.

Greenhouse-Geisser corrected p-values were reported when the sphericity assumption was violated in repeated measures designs. Error bars in error rates and subsequent analysis were expressed as $95 \%$ bias-corrected and accelerated (BCa) bootstrap confidence interval (CI) of the mean computed with 1000 iterations. This method takes into account the real distribution of the sample. Cohen's $d$ was used as the measure of effect size for $t$-tests.

\subsubsection{Phoneme analysis}

Since nearly $70 \%$ of the errors in both groups involved C1, phoneme resistance and phoneme confusions were computed only for $\mathrm{C} 1$. The phoneme $/ \mathrm{n} /$, which was presented in only one trial, was not included in these analyses. Phoneme error rates were calculated for each participant as the number of error for a particular phoneme related to the number of occurrences of the same phoneme. Statistical phoneme resistance to noise was assessed by comparing the percentage of error for each phoneme per participant to the phoneme percentage of occurrence. Thus by using one-sample two-sided $t$-tests we assessed if the percentage of error was higher or lower than expected or if there were no differences in resistance to noise. Scores in phoneme error rate were compared between groups with unpaired-sample $t$-tests. All the resulting $p$-values were false-discovery rate (fdr) corrected for multiple comparisons.

The reported phoneme confusion patterns include the total number of confusions between the studied French consonant phonemes in each group. Since the aim was to investigate differences between groups, the totals were not adjusted to the frequency of occurrence of each phoneme in the set of target words. For scale display purposes, we represented neither the number of times a phoneme was correctly reported nor the number of times a phoneme was omitted. Differences in phoneme confusions between the two groups were assessed with rank correlation analysis (Spearman's).

\section{Results}

The mean number of errors in word recognition in noise was higher in the dyslexic group (20.08\%) than in the control group of participants (16.51\%; See error rates and other descriptive measures of performance in Table 1). The difference in error rate between both groups (3.57\%) was statistically significant as assessed by a two-sided unpaired $t$-test (see the analysis of group differences in Table 1), thus supporting a deficit in word identification in noise in dyslexia. 
Table 1: Descriptive and inferential statistics of performance. The values under the dyslexics and control group are expressed as mean (95\% bias-corrected and accelerated bootstrap confidence interval).

\begin{tabular}{cccccccc}
\hline Measure & Dyslexics & Controls & $\boldsymbol{t}$ & df & $\boldsymbol{p}$ & $\mathbf{9 5 \%}$ CI diff & Cohen's $\boldsymbol{d}$ \\
\hline Error rate & $20.08(18.20-22.45)$ & $16.51(14.85-18.47)$ & 2.43 & 38 & .021 & $0.78-6.80$ & 0.77 \\
Substitution rate & $17.10(15.25-19.27)$ & $13.97(12.55-15.45)$ & 2.62 & 38 & .011 & $0.50-5.55$ & 0.83 \\
Omission rate & $2.98(1.89-4.24)$ & $2.54(1.32-3.90)$ & 0.41 & 38 & .693 & $-1.69-2.42$ & 0.13 \\
Errors profile & & & & & & & \\
\% substitution within errors & $86.15(79.92-91.30)$ & $86.41(79.00-93.10)$ & 0.057 & 38 & .962 & $-9.17-9.23$ & 0.02 \\
\% error involving C1 & $69.04(64.31-73.61)$ & $69.67(66.49-72.25)$ & 0.22 & 38 & .824 & $-6.38-5.25$ & 0.06 \\
\% errors involving C1 & $12.88(9.79-16.00)$ & $13.82(10.77-17.17)$ & 0.41 & 38 & .69 & $-5.51-3.49$ & 0.13 \\
\% errors involving V1 & $32.52(28.05-37.10)$ & $32.96(26.94-38.96)$ & 0.11 & 38 & .924 & $-8.33-7.44$ & 0.03
\end{tabular}

Errors were either substitutions or deletions. The majority of them were substitutions in both groups. Substitution rates were significantly higher in the dyslexic group (17.1\% vs. $14.0 \%)$, whereas omission rates were not (3.0\% vs. $2.5 \%)$. When comparing the profile of errors we did not find differences in the percentage of errors corresponding to substitutions (about $85 \%$ in both groups).

We tested whether error rates were modulated by the position in the first syllable. Figure 1 shows error rate for each position in the first syllable and for each group. Vowels show the lowest error rate. A mixed-model ANOVA with position (C1, C1' and V1) as within-subjects factor and group (dyslexic and control participants) as between-subjects factor showed a main effect of position $\left(F(2,76)=47.62, p<.001, \eta_{p}^{2}=.556\right)$. Post-hoc analysis indicated that error rate in $\mathrm{C1}$ ' was higher than error rate in C1 and V1 $(p=.01$ and $p<.001$ and 95\% CI of the difference [0.81-7.29 and 7.26-14.02] respectively). Similarly, error rate in C1 was higher than error rate in V1 ( $p<$ .001 , [5.66-7.52]). Neither the main effect of group nor the interaction between group and position of error were significant $\left(F(1,38)=2.02, p=.164, \eta_{p}^{2}=.050\right.$ and $F(2,76)=0.23, p=$ $\left..665, \eta_{p}^{2}=.006\right)$.

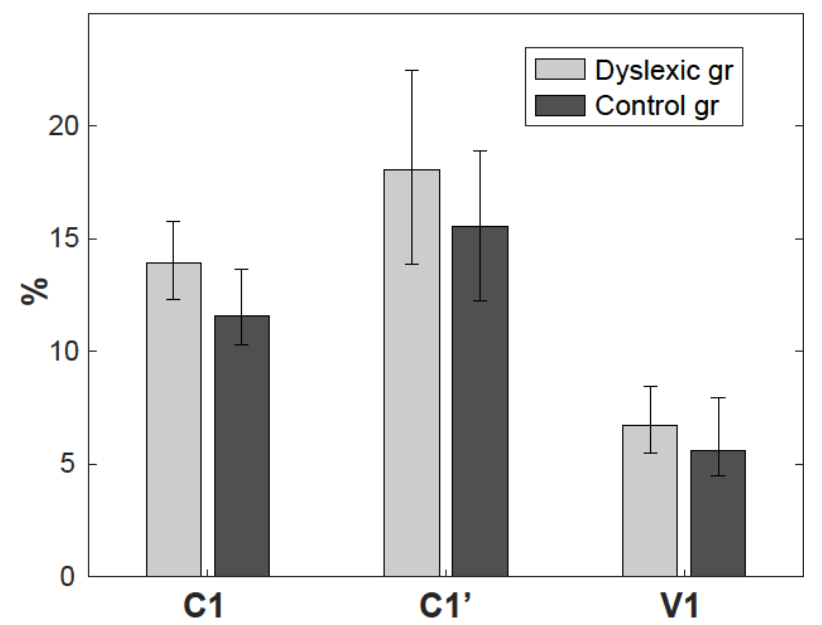

Figure 1: Error rate for each position in the first syllable (C1' indicates the second consonant of the cluster). Error bars depict 95\% BCa Bootstrap CI.

\subsection{Phoneme resistance to noise}

Despite the error rate being higher in C1', only 18 out of the 126 target disyllabic words were CCV. As a result, the majority of the observed errors in both groups consisted of $\mathrm{C} 1$ errors (the
$\%$ of errors involving $\mathrm{C} 1$ was close to $70 \%$, as shown in Table $1)$. Only $\mathrm{C} 1$ was therefore considered to investigate phoneme resistance and confusion. Figure 2 depicts error rate on phoneme identification for each C1 phoneme and for each group. The $\mathrm{C} 1$ phonemes have been sorted by the error rate ranking of resistance to noise in the dyslexic group. Phoneme resistance to noise was investigated by comparing the percentage of error for each phoneme per participant to the phoneme percentage of occurrence. For the control group, the ranking of resistance to noise, from the most resistant to the most vulnerable, according to its error rate, was:

$/ \mathrm{s} />/ \mathrm{g} />/ \mathrm{l} />/ \mathrm{k} />/ \mathrm{z} />/ \mathrm{s} />/ \mathrm{p} />/ \mathrm{b} />/ \mathrm{d} />/ \mathrm{g} />/ \mathrm{t} />/ \mathrm{m} />/ \mathrm{f} />/ \mathrm{v} /$

And for the dyslexic group:

$/ \mathrm{g} />/ \mathrm{s} />/ \mathrm{3} />/ \mathrm{k} />/ \mathrm{d} />/ \mathrm{l} />/ \mathrm{b} />/ \mathrm{g} />/ \mathrm{p} />/ \mathrm{b} />/ \mathrm{t} />/ \mathrm{f} />/ \mathrm{m} />/ \mathrm{v} /$

For both groups, the phonemes whose errors of identification was significantly below chance level were: /s/, / / $/$ and $/ \mathrm{k} /(p<$ fdr-adjusted critical $p$ value of $0.014, t(19)=13.44$, $d=3.01, t(19)=10.65, d=2.38, t(19)=2.81, d=0.63$ for the control group and $t(19)=6.78, d=1.52 \mathrm{for} / \mathrm{s} /, t(19)=13.31 d$ $=2.93$ for $/ \mathrm{f} /$ and $t(19)=2.81, d=0.63$ for the dyslexic group respectively). On the contrary, identification rates were significantly below chance level for $/ \mathrm{f} /, / \mathrm{m} /$ and $/ \mathrm{v} /$ for both groups ( $p<\mathrm{fdr}$-adjusted critical $p$ value of $0.014, t(19)=2.91$, $d=0.65$ for $/ \mathrm{f} /, t(19)=2.76, d=0.62$ for $/ \mathrm{m} /$ and $t(19)=3.44$, $d=0.77$ for $/ \mathrm{v} /$ for the control group of participants and $\mathrm{t}(19)=$ $2.84, d=0.63$ for $/ \mathrm{f} /, t(19)=2.69, d=0.60$ for $/ \mathrm{m} /, t(19)=2.85$, $d=0.64$ for $/ \mathrm{v} /$ for the dyslexic group).

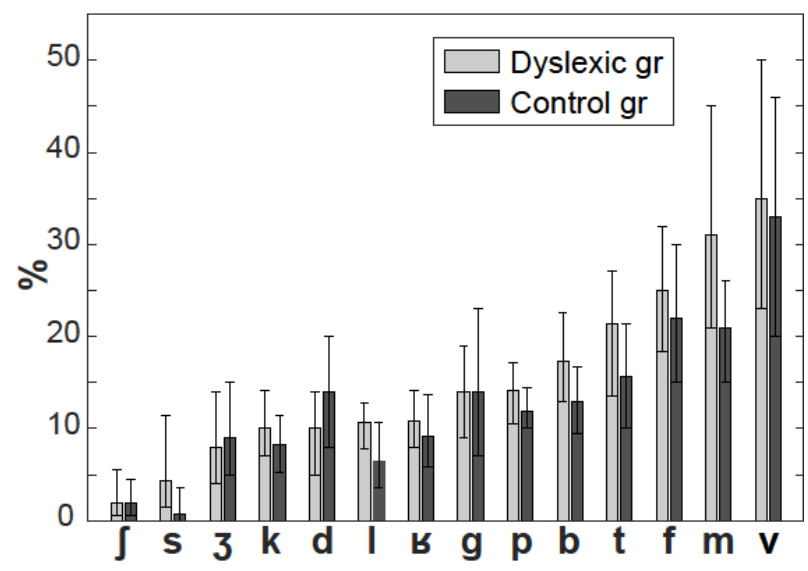

Figure 2: C1 phoneme error rate for each group.

When assessing differences in phoneme resistance between the dyslexic and the control group, no significant results were found for any phoneme type $(t<1.4, p>.05)$. 


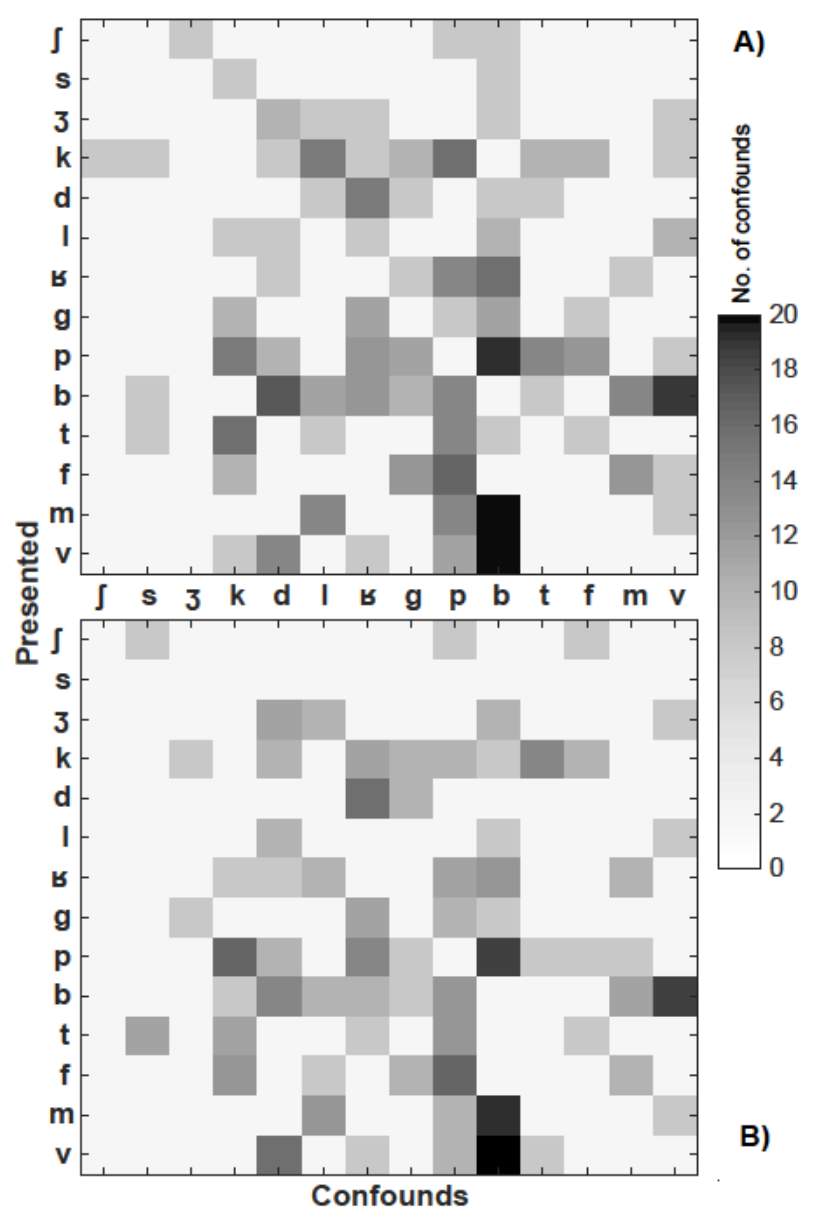

Figure 3: Confusion matrix for the dyslexic (A) and the control group of participants (B).

\subsection{Phoneme confusion matrices}

Figure 3 shows the confusion matrices for the dyslexic group (A) and the control group (B). Phonemes have been sorted by the observed ranking of phoneme resistance in dyslexic adults. The most common phoneme confusion pairs were the labials $/ \mathrm{b} /-/ \mathrm{v} /$ and $/ \mathrm{v} /-/ \mathrm{b} /$, the plosives $/ \mathrm{p} /-/ \mathrm{b} /$ and the labials $/ \mathrm{m} /-/ \mathrm{b} /$. Although the confusion matrix in the dyslexic group is more scattered, the two matrices are highly correlated (Spearman's $\rho=.735, p<.001,95 \%$ BCa CI [.628-.826]).

\section{Discussion}

The aim of this study was to investigate the existence and the scope of the speech perception in noise deficit in adults with dyslexia regarding: a) performance and b) the profile of phoneme resistance to noise and phoneme confusion. We found that error rates were higher in the group with dyslexia. On the other hand, we were not able to show qualitative differences in the profile of phoneme errors.

Overall, the observed pattern of phoneme errors replicates previous findings in healthy adults. As expected, vowels were more resistant than consonants $[7,11]$. The phonemes $/ \mathrm{s} /$ and $/ \mathrm{g} /$ were the most resistant consonants while $/ \mathrm{v} /, / \mathrm{m} /$ and $/ \mathrm{f} /$ were the most vulnerable, similarly to what have been reported previously in French (see [7] for a detailed interpretation of this result). The pattern of confusions was also consistent with previous studies in French but also in other languages (see /b/-
$/ \mathrm{v} /$ and $/ \mathrm{m} /-/ \mathrm{b} /$ confusions in English [8] or $/ \mathrm{p} /-/ \mathrm{b} /$ in Spanish [12]). Although we did not investigate all the French phonemes, it is not likely that differences are observed in the remaining C1 phonemes (i.e. $/ \mathrm{n} /$ and other less frequent $\mathrm{C} 1$ phonemes such as /x/, /j/, /z/).

Our data provide more behavioral evidence of a deficit in speech-in-noise perception in dyslexia using different type of stimuli. Compared to some previous studies $[13,14]$, the stimuli that were used had an ecological validity. Indeed, performance on discrimination between two phonemes or CV identification might overlook the deficit [15]. Other studies that examined the perception of whole sentences under noise [2] might allow the use of compensatory strategies. In our experiment we constrained context effects by using words presented in isolation and unrelated to each other.

In addition, we observed that the profile of errors for dyslexics is consistent with that of control participants. It has been proposed that phonological deficits in dyslexia might be due to poor phonological representation [16, 17] and we hypothesized that if this was the case, the pattern of errors would be different between the two groups of participants. Yet, we were not able to show differences in phoneme vulnerability to noise or in phoneme confusions between groups. Our data adds to a growing literature supporting the hypothesis of an intact phonological representation in dyslexia [6, 18, 19]. Our study should be replicated in a children population to ensure that there is not a phonological deficit in dyslexic children, which could be compensated in adulthood.

Taken together, our data are in accordance with the external noise exclusion hypothesis [20,21], which has been posited to account for reading and phonological deficits. It refers to the inability to exclude non-relevant noise. The quantitative differences observed between the two groups could be explained by the fact that adults with dyslexia have difficulties ignoring irrelevant background auditory information and therefore might have difficulties accessing phonological representations. This is in line with previous studies $[6,19]$ that posited that phonological representations are less accessible in dyslexia. In particular, the access and use of phonological representations would be more difficult in certain situations that engage conscious awareness, verbal short-term memory, or speeded access [19]. Our study shows that noise addition may be one of these situations.

\section{Conclusions}

This study provides new behavioral data supporting a deficit in speech-in-noise recognition in adults with dyslexia. Moreover, we characterized, for the first time to our knowledge, the profile of phoneme resistance and phoneme confusion when perceiving speech-in-noise. The quantitative decline in performance does not seem to be accompanied by differences in the profile of phoneme resistance to noise or phoneme confusions. This supports the hypothesis that the phonological representations are preserved in dyslexia. The perceptual processing deficit in dyslexia might be related to the inability to exclude irrelevant information.

\section{Acknowledgements}

We thank Nicolas Grimault for providing the background sound recordings and Marjorie Dole for her assistance on data collection. This research was supported by the European Research Council (starting-grant SpiN Project, n²09234). 


\section{References}

[1] F. Ramus et al., "Theories of developmental dyslexia: Insights from a multiple case study of dyslexic adults,” Brain, vol. 126, no. 4, pp. 841-865, 2003.

[2] A. R. Bradlow, N. Kraus, and E. Hayes, "Speaking Clearly for Children With Learning Disabilities,” J. Speech Lang. Hear. Res., vol. 46, no. 1, p. 80, Feb. 2003.

[3] J. C. Ziegler, C. Pech-Georgel, F. George, and C. Lorenzi, "Speech-perception-in-noise deficits in dyslexia," Dev. Sci., vol. 12, no. 5, pp. 732-745, 2009.

[4] S. Brady, D. Shankweiler, and V. Mann, "Speech perception and memory coding in relation to reading ability,” J. Exp. Child Psychol., vol. 35, no. 2, pp. 345-367, 1983.

[5] M. Dole, M. Hoen, and F. Meunier, "Speech-in-noise perception deficit in adults with dyslexia: Effects of background type and listening configuration," Neuropsychologia, vol. 50, no. 7, pp. 1543-1552, Jun. 2012.

[6] F. Ramus, "Neuroimaging sheds new light on the phonological deficit in dyslexia,” Trends Cogn. Sci., vol. 18, no. 6, pp. 274-275, 2014.

[7] J. Meyer, L. Dentel, and F. Meunier, "Speech Recognition in Natural Background Noise,” PLoS One, vol. 8, no. 11, p. e79279, Nov. 2013.

[8] D. L. Woods, E. W. Yund, T. J. Herron, and M. A. I. U. Cruadhlaoich, "Consonant identification in consonantvowel-consonant syllables in speech-spectrum noise," $J$. Acoust. Soc. Am., vol. 127, no. 3, pp. 1609-1623, Mar. 2010.

[9] M. D. Wang and R. C. Bilger, "Consonant confusions in noise: A study of perceptual features,” J. Acoust. Soc. Am. vol. 54, no. 5, pp. 1248-1266, 1973.

[10] B. New, C. Pallier, M. Brysbaert, and L. Ferrand, "Lexique 2: A new French lexical database," Behav. Res. Methods, Instruments, Comput., vol. 36, no. 3, pp. 516-524, 2004.

[11] L. Varnet, J. Meyer, M. Hoen, and F. Meunier, "Phoneme resistance during speech-in-speech comprehension," in 13th Annual Conference of the International Speech Communication Association, 2012.

[12] I. Moreno-Torres, P. Otero, S. Luna-Ramírez, and E. Garayzábal Heinze, "Analysis of Spanish consonant recognition in 8-talker babble,” J. Acoust. Soc. Am., vol. 141, no. 5, pp. 3079-3090, 2017.

[13] J. C. Ziegler, C. Pech-Georgel, F. George, and C. Lorenzi, "Noise on, voicing off: Speech perception deficits in children with specific language impairment,” J. Exp. Child Psychol., vol. 110, no. 3, pp. 362-372, 2011.

[14] V. Hazan, S. Messaoud-Galusi, and S. Rosen, "The Effect of Talker and Intonation Variability on Speech Perception in Noise in Children With Dyslexia,” J. Speech Lang. Hear. Res., vol. 56, no. 1, p. 44, Feb. 2013.

[15] V. Hazan, S. Messaoud-Galusi, S. Rosen, S. Nouwens, and B. Shakespeare, "Speech Perception Abilities of Adults With Dyslexia: Is There Any Evidence for a True Deficit?," J. Speech, Lang. Hear. Res., vol. 52, no. 6, pp. 1510-1529, Dec. 2009.

[16] R. Boada and B. F. Pennington, "Deficient implicit phonological representations in children with dyslexia," $J$. Exp. Child Psychol., vol. 95, no. 3, pp. 153-193, 2006.

[17] R. K. Wagner and J. K. Torgesen, "The nature of phonological processing and its causal role in the acquisition of reading skills.," Psychol. Bull., vol. 101, no. 2, pp. 192212, 1987.

[18] B. Boets et al., "Intact But Less Accessible Phonetic Representations in Adults with Dyslexia,” Science (80-. )., vol. 342, no. 6163, pp. 1251-1254, Dec. 2013.

[19] F. Ramus and G. Szenkovits, "What phonological deficit?," Q. J. Exp. Psychol., vol. 61, no. 1, pp. 129-141, Jan. 2008.

[20] R. L. Beattie, Z. L. Lu, and F. R. Manis, "Dyslexic adults can learn from repeated stimulus presentation but have difficulties in excluding external noise," PLoS One, vol. 6, no. 11, 2011.

[21] A. J. Sperling, Z. L. Lu, F. R. Manis, and M. S. Seidenberg,
"Deficits in perceptual noise exclusion in developmental dyslexia,” Nat. Neurosci., vol. 8, no. 7, pp. 862-863, 2005. 